Portland State University

PDXScholar

Physics Faculty Publications and Presentations

Physics

$12-1-2004$

\title{
The Meyer-Neldel rule for diodes in forward bias
}

\author{
Ralf Widenhorn \\ Portland State University \\ Michael Fitzgibbons \\ Erik Bodegom \\ Portland State University
}

Follow this and additional works at: https://pdxscholar.library.pdx.edu/phy_fac

Part of the Physics Commons

Let us know how access to this document benefits you.

\section{Citation Details}

Widenhorn, R., Fitzgibbons, M., \& Bodegom, E. (2004). The Meyer-Neldel rule for diodes in forward bias. Journal Of Applied Physics, 96(12), 7379-7382.

This Article is brought to you for free and open access. It has been accepted for inclusion in Physics Faculty Publications and Presentations by an authorized administrator of PDXScholar. Please contact us if we can make this document more accessible: pdxscholar@pdx.edu. 


\title{
The Meyer-Neldel rule for diodes in forward bias
}

\author{
Ralf Widenhorn, ${ }^{\text {a) }}$ Michael Fitzgibbons, and Erik Bodegom ${ }^{\text {b) }}$ \\ Department of Physics, Portland State University, Portland, Oregon 97207-0751
}

(Received 14 June 2004; accepted 21 September 2004)

\begin{abstract}
We analyzed the temperature dependence of the forward current of a silicon diode. Instead of representing the data in the ordinarily used current versus voltage graph, the currents are plotted for different voltages as a function of the inverse temperature. The constant voltage curves can be fitted linearly and the extrapolations of the fits seem to merge to one common focal point. Hence, we demonstrate that a real diode follows the Meyer-Neldel rule (MNR). It is shown that the MNR is due to a shift of the current from ideal-diode to high-injection-diode behavior. We will argue that the merging of the different Arrhenius plots toward one focal point, and hence a MNR, can be the result of various mechanisms. The general requirements to observe a MNR are not very restrictive. It is therefore not surprising that the MNR has been observed in a multitude of systems. The origin that gives rise to the MNR can be manifold and allows for different models to explain its occurrence. (C) 2004 American Institute of Physics. [DOI: 10.1063/1.1818353]
\end{abstract}

\section{INTRODUCTION}

Thermally activated processes frequently follow an exponential power law of the form,

$$
X=X_{0} \exp (-\Delta E / k T) .
$$

To extract the exponential prefactor $X_{0}$ and the activation energy $\Delta E$ from an experimental data set, one fits the natural logarithm of $X$ linearly versus the inverse temperature $T^{-1}$. Graphically the activation energy is found as the negative of the slope of the plot of $\ln (X)$ versus $T^{-1}$, the so-called Arrhenius plot. For related thermally activated processes the activation energy can vary from sample to sample. Meyer and Neldel found in 1937 that the activation energy and the natural logarithm of the exponential prefactor for related samples are frequently linearly related, ${ }^{1}$

$$
\ln \left(X_{0}\right)=\ln \left(X_{00}\right)-\frac{\Delta E}{E_{\mathrm{MN}}},
$$

where $X_{00}$ and $E_{\mathrm{MN}}$ are constants. For positive $E_{\mathrm{MN}} \mathrm{S}$ this relationship is known as the Meyer-Neldel rule (MNR). The less frequently observed cases with negative $E_{\mathrm{MN}}$ values are referred to as inverse or anti-Meyer-Neldel rule. ${ }^{2-5}$ MNRlike behavior has been found in various different systems. Frequently MNR behavior is reported for electrical properties such as the conductivity or for diffusion measurements. This raises the question if there is one underlying mechanism that causes the seemingly universal occurrence of the MNR. The debate about the physical significance of the MNR is not settled as of yet. One common explanation is the statistical shift model, which describes the MNR as the consequence of the change of the Fermi level with temperature. ${ }^{6}$ Others argue the MNR arises for processes for which the total energy is provided by multiple excitations. ${ }^{7-10}$ We have shown that

\footnotetext{
${ }^{a)}$ Electronic mail: ralfw@pdx.edu

b) Present address: Electronic Instrumentation Laboratory, Delft University of Technology, 2628 CD Delft, The Netherlands; electronic mail: bodegom@pdx.edu
}

multiple processes coexisting at the same time can also lead to the MNR. ${ }^{11}$ This explanation requires slightly positive curvatures of the Arrhenius plot and result in $E_{\mathrm{MN}}$ values close to the thermal energy at which the experiment was measured. Nonlinear Arrhenius plots are common ${ }^{12-21}$ and it has been pointed out that frequently a correlation between $E_{\mathrm{MN}}$ and the experimental temperature range is found. ${ }^{22-24}$

To get a better understanding of implications of the MNR it is useful to substitute the value of $X_{0}$ as given by the MNR [Eq. (2)] into the Arrhenius law [Eq. (1)]:

$$
X=X_{00} \exp \left(\Delta E\left[\frac{1}{E_{\mathrm{MN}}}-\frac{1}{k T}\right]\right) .
$$

Since the activation energy has positive a value, the exponent of Eq. (3) is negative for temperatures smaller than the socalled isokinetic temperature $T_{\mathrm{MN}}=E_{\mathrm{MN}} / k$. Hence, for $T<T_{\mathrm{MN}}$, $X$ will be larger for samples with smaller activation energies. At $T=T_{\mathrm{MN}}, X$ will be independent of the activation energy and will become smaller for samples with larger activation energies at $T>T_{\mathrm{MN}}$. Most experiments show data at temperatures smaller than the isokinetic temperature. For such cases the Arrhenius plots of samples with different activation energies converge with increasing temperature toward one "focal" point at $T=T_{\mathrm{MN}}$.

We will argue that the convergence of the linear fits to the data in the Arrhenius plot is quite natural for a multitude of systems. The underlying mechanism and therefore the explanation of the occurrence of the MNR might vary from system to system. We will present data of the $I V$ characteristic of a diode in forward bias. Diodes, the fundamental building block of every semiconductor circuit, have been studied extensively. ${ }^{25,26}$ Our experimental data set in itself is not novel but the analysis in terms of the MNR gives a different perspective on the origin of the MNR.

\section{IDEAL DIODE}

The first step necessary to understand the operation of any semiconductor device is the analysis of a simple $p n$ 
junction, the interface between a positively and negatively doped semiconductor. If one applies a voltage $V$ to a $p n$ junction, the current $I$ versus voltage characteristics is generally approximated by the well-known diode equation,

$$
\begin{aligned}
I= & I_{\text {gen }}[\exp (q V / k T)-1]=q A\left[\frac{D_{p} n_{i}^{2}}{L_{p} N_{D}}+\frac{D_{n} n_{i}^{2}}{L_{n} N_{A}}\right] \\
& \times[\exp (q V / k T)-1],
\end{aligned}
$$

where $q$ is the charge of an electron and $k$ is Boltzmann's constant. The reverse generation current $I_{\text {gen }}$ depends on the cross section of the junction $A$, the diffusivity $D$, the diffusion length $L$, the intrinsic electron concentration $n_{i}$, and the donor and acceptor doping level $N_{D}$ and $N_{A}$. The $p$ subscripts represent the values for holes and the $n$ subscripts stand for electrons. For the sake of simplicity we assume that the doping concentration on one side of the junction is much larger than for the other side. For example, for a $p^{+} n$ structure, i.e., a high acceptor concentration, and $q V \gg k T$ the diode equation can be simplified to

$$
I=q A \frac{D_{p} n_{i}^{2}}{L_{p} N_{D}} \exp (q V / k T) .
$$

Both $D_{p}$ and $L_{p}$ are slightly temperature dependent, but the $T$ dependence of $I$ is mainly determined by $n_{i}$ and the exponential function in Eq. (5). The intrinsic carrier concentration $n_{i}$ for a semiconductor with band gap $E_{g}$ is given by

$$
n_{i}^{2}=N_{c} N_{v} \exp \left(E_{g} / k T\right) .
$$

The number of states in the valence band $N_{v}$ and the number of states in the conduction band $N_{c}$ is again slightly temperature dependent. However, the temperature dependence of $n_{i}$ is dominated by the exponential function. Hence, the $T$ dependence for the forward current through the junction can be expressed as

$$
I \sim \exp \left(-\frac{E_{g}-q V}{k T}\right) .
$$

The large panel in Fig. 1 shows the Arrhenius plots for an ideal diode under the above described conditions. The data was calculated for an assumed band gap of $1.12 \mathrm{eV}$ at several different voltages. This band gap corresponds to the band gap of silicon and is assumed temperature independent. The prefactor is arbitrary but independent of $T$ and $V$. One can see that the curves satisfy the basic MNR conditions of linearity and a common focal point. The activation energy for each of the lines is given by

$$
\Delta E=E_{g}-q V .
$$

Plotting the activation energy versus the natural logarithm of the prefactor results in a linear line as predicted by the MNR (see small panel in Fig. 1).

It is obvious that the ordinate values of the plot are independent of the activation energy and equal the logarithm of the constant prefactor $I_{0}$. The resulting characteristic energy or temperature is given by $E_{\mathrm{MN}}=k T_{\mathrm{MN}}=\infty$. This result is in many ways trivial, but it sheds more light on the MNR. The requirement to observe a nontrivial MNR is the convergence to a focal point away from $T^{-1}=0$. For lines converg-

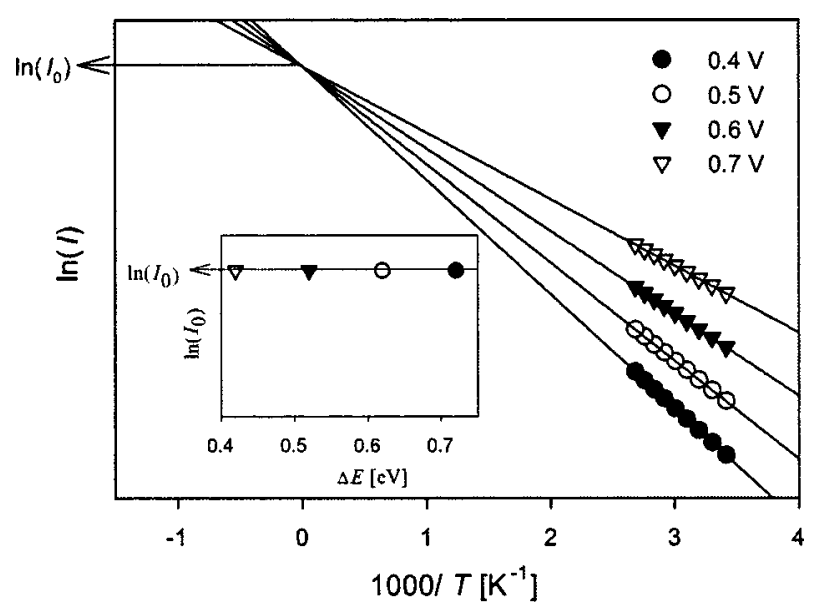

FIG. 1. Computed Arrhenius plot for an ideal diode. The band gap is assumed to be $1.12 \mathrm{eV}$ and data points are calculated for every $10 \mathrm{~K}$ between 293 and $373 \mathrm{~K}$ for an arbitrary prefactor $I_{0}$. The small panel displays the MNR plot, the logarithm of the prefactor vs the activation energies for the different voltages.

ing at positive abscissa values the regular MNR results; if the convergence is slow and the focal point is at negative $T^{-1}$ values the less common anti-MNR or inverse MNR is observed. To answer the question what causes the MNR one must answer the question what causes a convergence away from $T^{-1}=0$.

The actual temperature dependence of the $I V$ characteristic differs from the idealized description of diode as outlined above. We will show how such deviations can lead to an earlier convergence of the different Arrhenius plots and hence to a "real" MNR.

\section{REAL DIODE}

For a given temperature and variable voltages, the current can be separated into different regimes. ${ }^{26}$ In the low voltage regime, the generation and recombination current describes the $I V$ characteristic. For high voltages, a large number of carriers are injected into the semiconductor and the diode again behaves nonideal. In both of these cases the current is characterized by

$$
I \sim \exp \left(-\frac{E_{g}-q V}{2 k T}\right) .
$$

The activation energy in these regions of the $I V$ plot is only half of the activation energy of the ideal diode:

$$
\Delta E=\frac{E_{g}-q V}{2 k T} .
$$

Only in the region between generation-recombination current and high injection current does the diode behave like an ideal diode. For very high voltages, the series resistance of the silicon causes the curves to deviate even more from the ideal behavior. Empirically the current of a diode is often expressed in the form, 


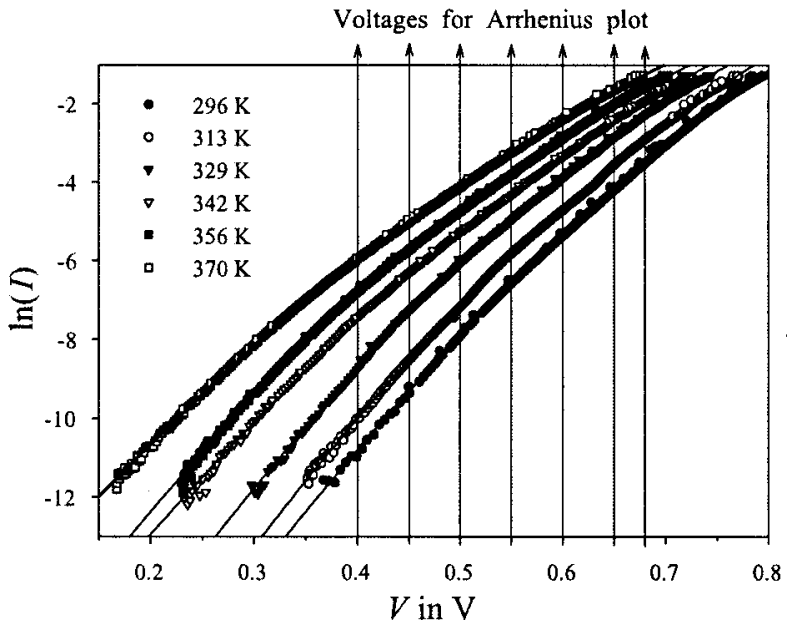

FIG. 2. Experimental data for $\ln (I)$ vs $V$ for six different temperatures between 296 and $370 \mathrm{~K}$.

$$
I \sim \exp \left(-\frac{E_{g}-q V}{n k T}\right)
$$

The empirical parameter $n$ is often called the ideality factor. Its value is equal to 1 for an ideal diode and equal to 2 in the generation-recombination region and in the high-injection region. In the transition region, its value is between 1 and 2 .

We used a GI502 general purpose rectifier diode manufactured by General Semiconductors to measure the $I V$ curves at six different temperatures (between 296 and $370 \mathrm{~K}$ ). The diode was submerged in a oil bath which was kept at approximately constant temperatures for each data set. The plastic cover of the diode was removed such that the junction was in intimate contact with the oil. The temperature was constantly monitored with a Lake Shore DT-470 diode temperature sensor. The current and voltage pairs shown in Fig. 2 were measured with a Hewlett-Packard 34970 A data acquisition unit. For each temperature, the voltage was slowly swept from its minimum to its maximum voltage. The slight wobbles in the data are due to small temperature fluctuations. In the following section the correct temperatures are used to investigate the MNR. The lower voltage limit was determined by the precision of the current probe.

For an ideal diode according to Eq. (5), one would expect a slope of $q / k T$ for the different $I V$ plots. A diode in the high-injection region would show a slope of $q / 2 k T$. One sees immediately that the curves in the figure do not follow a straight line. Therefore, we added a quadratic best fitting line through the data sets. The derivative of the quadratic fit can be used to calculate the slope at a given voltage. The ideality factor can be calculated from the general expression of the slope: $q / n k T$. For example, at $296 \mathrm{~K} n$ changes from roughly 1.1 at $0.4 \mathrm{~V}$ to 1.9 at $0.678 \mathrm{~V}$. At $370 \mathrm{~K}, n$ increases from $\approx 1.4$ at $0.4 \mathrm{~V}$ to 2.2 at $0.678 \mathrm{~V}$. Hence, at low voltages or better at low current injection, the device behaves close to an ideal diode. With increasing current, the diode reaches the high-injection regime and the ideality factor increases to $n$

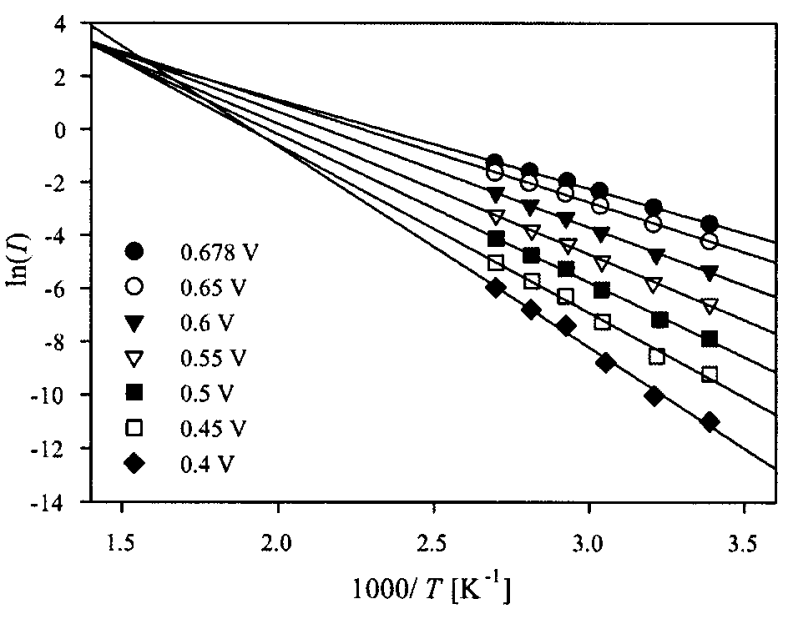

FIG. 3. Arrhenius plot for a real diode. The best linear fits to the experimental data seem to converge to a common focal point.

$=2$. At high voltage and high temperatures $n>2$, an indication that the series resistance of the silicon causes a potential drop outside the junction region.

\section{MEYER-NELDEL RULE FOR A DIODE}

To obtain the Arrhenius plot for a real diode, we plotted the $\ln (I)$ values for a given voltage. Each data set can be found by drawing a vertical line through the $I V$ curves shown in Fig. 2. Figure 3 shows the values for the current and the actual measured temperature, not the average temperature as in Fig. 2. For a given voltage, the data are fitted linearly according to

$$
\ln (I)=\ln \left(I_{0}\right)-\frac{\Delta E}{k T} .
$$

One can see that the individual $\ln (I)$ and $\Delta E$ pairs, shown in Fig. 4, lie approximately on a straight line. The characteristic energy $E_{\mathrm{MN}}$ as slope of the linear fit in Fig. 4 is given by $56.5 \mathrm{meV}$. The isokinetic temperature $T_{\mathrm{MN}}=E_{\mathrm{MN}} / k$ is equal to $657 \mathrm{~K}$. The focal point in Fig. 3 is therefore located at $1.52 \times 10^{-3} \mathrm{~K}^{-1}$. Unlike in the case of an ideal diode the MNR is nontrivial with an common focal point at positive

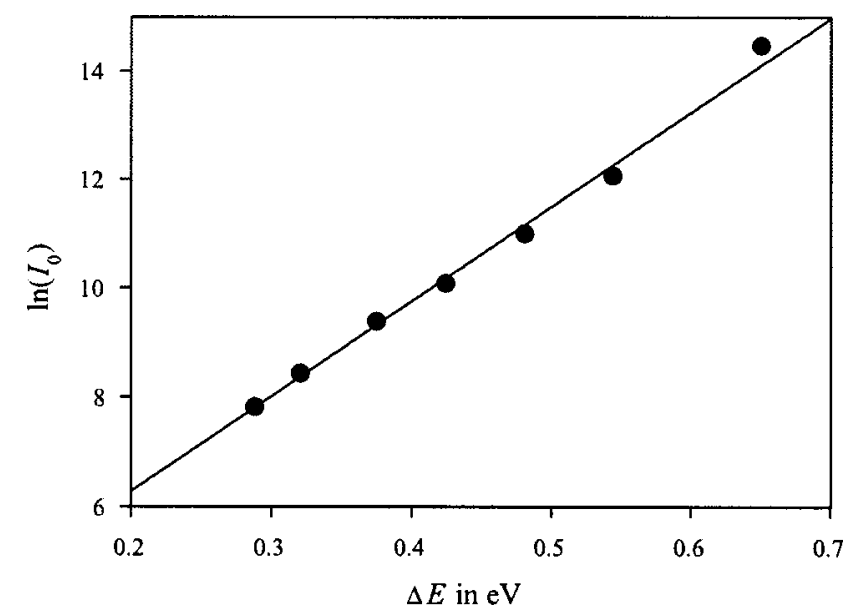

FIG. 4. Meyer-Neldel rule plot for a real diode. The linear fit yields an isokinetic temperature of $657 \mathrm{~K}$. 
TABLE I. Activation energies and ideality factors for the different voltages.

\begin{tabular}{llll}
\hline \hline$V(\mathrm{~V})$ & $E_{g}-q V(\mathrm{eV})$ & $\Delta E(\mathrm{eV})$ & $n$ \\
\hline 0.678 & 0.442 & 0.288 & 1.53 \\
0.65 & 0.47 & 0.32 & 1.47 \\
0.6 & 0.52 & 0.375 & 1.39 \\
0.55 & 0.57 & 0.424 & 1.34 \\
0.5 & 0.62 & 0.481 & 1.29 \\
0.45 & 0.67 & 0.544 & 1.23 \\
0.4 & 0.72 & 0.650 & 1.11 \\
\hline \hline
\end{tabular}

abscissa. To answer the question of what causes the lines to converge earlier than for the ideal diode, one needs to take a closer look at each line in the Arrhenius plot. For an ideal diode, the slope at each voltage can be calculated with Eq. (8). For a real diode one finds from Eq. (11) that

$$
\Delta E=\frac{E_{g}-q V}{n k T} .
$$

Comparing the ideal activation energies calculated with Eq. (8) and the activation energies obtained from the slope in Fig. 3, the ideality factor as described in Eq. (13) can be calculated for the different voltages (see Table I). At any given temperature the current for larger voltages is higher and therefore closer to the high-injection regime with $n=2$. As one can see in Fig. 2, the plots curve downward with increasing voltage. Hence, the current is lower than one would expect for an ideal diode. This saturation effect causes the Arrhenius plots to move closer together and the lines to converge well before $T^{-1}=0 \mathrm{~K}^{-1}$. In other words, the MNR is due to the fact that the transition to the high-injection regime occurs at lower temperatures for larger voltages.

A closer examination of the Arrhenius plot shows that the focal point of the real diode is not perfect. Especially the plot for $0.4 \mathrm{~V}$ crosses the other curves at lower temperatures than $T_{\mathrm{MN}}$. For example, the intercept with the $0.45 \mathrm{~V}$ line at $T=500 \mathrm{~K}$ is significantly different from the isokinetic temperature. This deviation is also seen in the far right data point in the Meyer-Neldel plot (Fig. 4). However, the MeyerNeldel plot is still reasonably linear. Generally, one can say that even a low quality focal point in the Arrhenius plot can lead to an acceptable linear Meyer-Neldel plot.

This example of the diode fits a whole class of cases for which one would expect MNR-like behavior. The conditions required to observe a MNR are in fact quite natural. At low temperatures, the process with the smaller activation energy happens at a faster rate. The different activation energies make it already eminent that the lines for different samples converge with increasing temperature. The key requirements are that they converge close to one point that is not at $T^{-1}$ $=0 \mathrm{~K}^{-1}$. Saturation effects can cause curves to tilt toward an apparent common maximum rate. This maximum rate is only apparent and might not always be of physical significance.

\section{CONCLUSION}

We demonstrated that the well-understood system of a diode in forward bias shows the Meyer-Neldel rule. The occurrence of the MNR can be explained by an earlier transition to the high-injection regime at large voltages. Unlike in the model for the MNR for the dark current in a chargecoupled device, ${ }^{27}$ the Arrhenius plots presented here are linear. Instead of two coexisting processes in that case, the MNR for diodes is the result of different $I V$ characteristics in different current regimes. We would expect that the MNR in other systems can be explained by a similar analysis. The multitude of systems that show the MNR and the numerous explanations for it suggests indeed that its occurrence is a result of different mechanisms. Our analysis shows that the requirement of converging lines is in fact quite natural and can be met in many ways. Hence, the very common discovery of the MNR.

\section{ACKNOWLEDGMENT}

This work was supported by the Murdock Foundation through the Partners in Science program.

${ }^{1}$ W. Meyer and H. Neldel, Z. Tech. Phys. (Leipzig) 12, 588 (1937).

${ }^{2}$ R. Flückiger, J. Meier, M. Goetz, and A. Shah, J. Appl. Phys. 77, 712 (1995).

${ }^{3}$ H. Meiling and R. E. I. Schropp, Appl. Phys. Lett. 74, 1012 (1999).

${ }^{4}$ G. Lucovsky, C. Wang, M. J. Williams, Y. L. Chen, and D. M. Maher, Mater. Res. Soc. Symp. Proc. 283, 443 (1993).

${ }^{5}$ A. Rubino et al., Mater. Res. Soc. Symp. Proc. 297, 509 (1993).

${ }^{6} \mathrm{H}$. Overhof and P. Thomas, Electronic Transport in Hydrogenated Amorphous Semiconductors (Springer, Berlin, 1989).

${ }^{7}$ A. Yelon, B. Movaghar, and H. M. Branz, Phys. Rev. B 46, 12244 (1992).

${ }^{8}$ A. Yelon and B. Movaghar, Phys. Rev. Lett. 65, 618 (1990).

${ }^{9}$ G. Boisvert, L. J. Lewis, and A. Yelon, Phys. Rev. Lett. 75, 469 (1995).

${ }^{10}$ A. Yelon and B. Movaghar, Appl. Phys. Lett. 71, 3549 (1997).

${ }^{11}$ R. Widenhorn, A. Rest, and E. Bodegom, J. Appl. Phys. 91, 6524 (2002).

${ }^{12}$ D. H. Tassis, C. A. Dimitriadis, and O. Valassiades, J. Appl. Phys. 84, 2960 (1998).

${ }^{13}$ K. Morii, T. Matsui, H. Tsuda, and H. Mabuchi, Appl. Phys. Lett. 77, 2361 (2000).

${ }^{14}$ C. Guillén and J. Herrero, J. Appl. Phys. 71, 5479 (1992).

${ }^{15}$ K. Herz and M. Powalla, Appl. Surf. Sci. 91, 87 (1995).

${ }^{16}$ M. Bruzzi et al., Eur. Phys. J. B 36, 3 (2003).

${ }^{17}$ D. H. Tassis, C. A. Dimitriadis, J. Brini, G. Kamarinos, and A. Birbas, J. Appl. Phys. 85, 4091 (1999).

${ }^{18}$ D. G. Papageorgiou and G. A. Evangelakis, Surf. Sci. 461, L543 (2000).

${ }^{19}$ E. Quartarone, P. Mustarelli, C. Tomasi, and A. Magistris, J. Phys. Chem. B 102, 9610 (1998).

${ }^{20}$ J. C. Wang and Y. F. Chen, Appl. Phys. Lett. 73, 948 (1998).

${ }^{21}$ P. A. W. E. Verleg and J. I. Dijkhuis, Phys. Rev. B 58, 3917 (1998).

${ }^{22}$ D. J. Dunstan, Solid State Commun. 107, 159 (1998).

${ }^{23}$ R. Kirchheim and X. Y. Huang, Phys. Status Solidi B 144, 253 (1987).

${ }^{24}$ P. Mialhe, J. P. Charles, and A. Khoury, J. Phys. D 21, 383 (1988).

${ }^{25}$ A. S. Grove, Physics and Technology of Semiconductor Devices (Wiley, New York, 1967).

${ }^{26}$ S. M. Sze, Physics of Semiconductor Devices, 2nd ed. (Wiley, New York, 1981).

${ }^{27}$ R. Widenhorn, L. Mündermann, A. Rest, and E. Bodegom, J. Appl. Phys. 89, 8179 (2001). 